\title{
La política de vivienda en Europa. Consideraciones desde la perspectiva de los Servicios Sociales de interés general
}

\author{
Housing policy in Europe. Factors of general interest \\ from the Social Services perspective \\ Eva María JuAn TOSET \\ Universidad de Granada \\ evam.juan@gmail.com
}

Recibido: 06/11/2011

Revisado: 09/11/2011

Aceptado: 17/12/2011

Disponible on line: 03/08/2012

\begin{abstract}
Resumen
Este artículo intenta aproximarse a la cuestión de la política del sector social de la vivienda en la Unión Europea. Se aborda el reconocimiento de la vivienda social como servicio social de interés general y su papel en la consecución de la cohesión social y territorial, así como los retos que debe afrontar la política de vivienda en la actual coyuntura económica y financiera. Se analiza brevemente la tendencia europea en materia política de vivienda social y las dificultades de los modelos de gestión utilizados en los Estados miembros de la Unión Europea.
\end{abstract}

Palabras clave: Política Social, Política de Vivienda, Servicios Sociales, Trabajo Social, Unión Europea.

\begin{abstract}
This article seeks to address the matter of social sector housing policies in the European Union. It deals with the recognition of social housing as a social service of general interest and its role in achieving social and territorial cohesion, as well as with the challenges facing housing policy in the current economic and financial environment. Briefly discusses the European trend in social housing policy and the difficulties of the management models used in the Member States of the European Union.
\end{abstract}

Key words: Social Policy, Housing Policy, Social Services, Social Work, European Union.

Referencia normalizada: Juan Toset, E. M. (2012): «La política de vivienda en Europa. Consideraciones desde la perspectiva de los Servicios Sociales de interés general». Cuadernos de Trabajo Social, 25(2): 451-459.

Sumario: Introducción. 1. El contexto europeo de las políticas de vivienda. el papel de las viviendas sociales en los servicios sociales de interés general. 2. El sector social de la vivienda en Europa. 3. Algunos retos para la política de viviendas sociales en Europa. 4. Conclusiones. 5. Referencias bibliográficas.

\section{Introducción}

Los Servicios Sociales de interés general reconocen, en la perspectiva europea, aquellos servicios esenciales para los ciudadanos que contribuyen a una mayor cohesión social y colaboran en alcanzar un modelo social europeo. La inmersión de la vivienda social como servicio social de interés general supone avanzar hacia una mirada que contempla su dimensión social pero, además, la sitúa en una situación compleja, teniendo en cuenta las tendencias en materia de política de vivienda llevada a cabo en los países europeos desde los años 1980 y con marcadas diferencias en los modelos de gestión.

Si bien esta heterogeneidad ha sido una constante desde el final de la Segunda Guerra Mundial, la situación actual de crisis financiera y económica, el retroceso de la intervención pública o la ralentización en la implementación de 
políticas sociales, es una constante en la mayoría de los países de la Unión Europea que afecta, entre otros servicios, a la vivienda social.

El alquiler social, o de promoción pública, tradicionalmente observado por los estados como una solución habitacional para las personas con menores ingresos económicos, fundamentalmente en los países de la Europa del Sur, ha comenzado a retraerse y ser objeto de debate. En este sentido, el interés de los gobiernos por resolver modelos de gestión inadecuados en las viviendas sociales (y sus consecuencias adversas para el equilibrio de las ciudades), ha precisado de intervenciones en muchos países, destinadas a paliar, principalmente, situaciones de exclusión social con una dimensión territorial importante. Barrios desfavorecidos, parques de viviendas sociales degradadas o cascos históricos con niveles de deterioros importantes, están siendo objeto de actuaciones destinadas a minimizar procesos de segregación espacial o condiciones de habitabilidad inadecuadas entre una parte importante de sus residentes. Dichas actuaciones, elaboradas desde estrategias político/económicas diversificadas, dependiendo el país donde se sitúen, no pueden obviar el contexto europeo en el que nos emplazamos ni, tampoco, las tendencias en materia de política de vivienda social que están consolidándose en, cada vez más, un menor intervencionismo público.

Con este objetivo se aborda el contenido del presente artículo. Con la finalidad de presentar la situación del sector público de viviendas sociales en relación a las tendencias en materia de políticas de vivienda que, de una manera $u$ otra, dibujan las líneas estratégicas de intervención. Para ello exponemos una reflexión sobre la concepción de los diferentes modelos de gestión ligados a las políticas de los estados miembros y las consecuencias que tienen en la actualidad para el sector de la vivienda social. Introduciremos algunas de las principales consideraciones que vinculan la vivienda social con los Servicios Sociales de interés general de carácter no económico a partir de la denominada Estrategia de Lisboa y la Agenda Social. Para finalizar con un breve análisis sobre las tendencias en la política de vivienda y los retos, que para la política social y los Servicios Sociales, posee la vivienda social como servicio esencial para los ciudadanos.

\section{El contexto europeo de las políticas de vi- vienda. El papel de las viviendas sociales en los Servicios Sociales de interés general}

La intervención de la Unión Europea en materia social encuentra su base legal en el Título $\mathrm{X}$ de la versión consolidada del Tratado de Funcionamiento de la Unión Europea. Pero no será hasta el Consejo Europeo de Lisboa, cuando se emprenda una estrategia en la lucha contra la pobreza en el marco de un desarrollo económico sostenible, creación de empleo y cohesión social. A partir de la Cumbre de Lisboa, el enfoque de la política social de la Unión Europea se concentra en una coordinación reforzada destinada a estimular reformas estructurales en los modelos de protección social.

La coordinación de las políticas contra la pobreza comienza a percibirse desde una perspectiva de coordinación de diferentes acciones destinadas a promover la cohesión social y así, se integran elementos que, hasta el momento, habían sido percibidos de forma sectorializada como, por ejemplo, la política de vivienda y su papel en la satisfacción de necesidades sociales emergentes. En este sentido, desde las instituciones de la Unión Europea se reconoce, por ejemplo, que las buenas condiciones de la vivienda son necesarias para la cohesión social. Así, cada vez más, la política de vivienda se observa como un mecanismo de intervención social que debe integrarse con otras políticas en los ámbitos de la salud, la educación, la regeneración urbana o los servicios sociales. Sin embargo, la heterogeneidad de tendencias en la intervención pública sobre estas políticas, la diversidad de modelos de gestión y las particularidades de la situación de cada Estado miembro de la Unión Europea, hace complejo un análisis lineal de este contexto en materia de política de vivienda.

Este esfuerzo por integrar elementos que refuercen las transformaciones en los sistemas de protección social hacia la modernización y con una perspectiva de integralidad, afectó, asimismo, al análisis de la vivienda social como factor fundamental en el desarrollo sostenible y la lucha contra la exclusión social. Llevando a considerarla como servicio social de interés general de carácter no económico.

A pesar de que las competencias en política de vivienda corresponden a los estados miembros, la vivienda social comienza a contem- 
plarse como un servicio de interés general que resulta relevante, como hemos señalado, en los procesos de cohesión social o en la lucha contra la pobreza. Un bien que debe considerarse accesible y de calidad en todo el territorio de la Unión Europea, una parte esencial de la ciudadanía europea, necesario para disfrutar plenamente de sus derechos universales (COM, 2004).

En el Libro Verde sobre los servicios de interés general (SIG), la vivienda social se incluye dentro de los servicios sociales de interés general (SSIG), con un papel concreto a desempeñar, junto con la asistencia sanitaria, los cuidados de larga duración o la seguridad social, integrada en el modelo europeo de sociedad y contemplada por el Consejo de Europa de Lisboa en marzo de 2000 (COM, 2004).

Los Servicios Sociales de interés general en la Unión Europea se diferencian en dos grandes grupos. Por un lado, aquellos sistemas legales y sistemas complementarios de protección social que cubren riesgos relacionados con la salud, la vejez o la discapacidad, entre otros (COM, 2006). Por el otro, aquellos servicios sociales esenciales con una función preventiva y de cohesión social que aportan una intervención facilitadora de la inclusión social, y garantizan la realización de los derechos fundamentales. Éstos servicios esenciales incluirían la ayuda a los ciudadanos para afrontar retos inmediatos de la vida; las actividades destinadas a la adquisición de competencias para su inserción en la sociedad y en el mercado laboral; aquellas actividades a garantizar la inclusión de las personas con necesidades de cuidados a largo plazo y, por último, la vivienda social «que permite el acceso a la vivienda a las personas con escasos ingresos» (COM, 2006, p. 11).

Además de incluirse la vivienda social como un servicio social de interés general, ésta también se ha contemplado como elemento de análisis en documentos que versan sobre el desarrollo urbano integrado. La «Carta de Leipzig sobre Ciudades Europeas Sostenibles» (2007) plantea, entre otras cuestiones, la renovación del parque de viviendas como elemento favorecedor de la mejora de la calidad de vida de sus residentes, o el planteamiento de unas políticas sociales de vivienda como instrumento facilitador de la cohesión e integración social en las ciudades, contribuyendo a la estabilidad de los barrios.

Un enfoque integrado del desarrollo urbano sostenible, basado en la armonización de las políticas públicas que afectan a la ciudad y que contempla la interacción del medioambiente, la economía y la cuestión social como pilares fundamentales. Un planteamiento que evidencia la necesidad de políticas que venzan las tradicionales propuestas sectoriales (Apéndice a la declaración final de los ministros responsables de desarrollo urbano para la implementación de la Carta de Leipzig, 2008).

Como ejemplo de la necesidad de observar la vivienda social como principio integrador, en el Informe conjunto sobre Protección e Inclusión Social (2009), la vivienda aparece como medida inclusiva en relación a la población romaní y su situación de exclusión, el fenómeno de las personas sin hogar, las desigualdades sanitarias entre los diferentes grupos socioeconómicos o la exclusión financiera debida a la actual crisis económica (pp. 7503/7509).

Sin embargo, la situación de los servicios sociales de interés general y el de la política social de vivienda, entre otros, se encuentra en un momento complejo. En 2009, Proinsias De Rossa (2009), en su Informe sobre el Futuro de los servicios sociales de interés general (INI, pp. 2009-2222,), ya instaba a los Estados miembros a mantener la misma disponibilidad de servicios accesibles, garantizando un acceso no discriminatorio e insistiendo en evitar que la crisis financiera y económica ponga en peligro el desarrollo de estos servicios.

Además, la mayoría de los estados miembros no contemplan la vivienda social dentro de las prestaciones de responsabilidad pública, lo que continúa manteniendo este servicio fuera de los recursos existentes en los servicios sociales a pesar que, cada vez más, se trate de una necesidad social no cubierta por el mercado (Tornos y Galán, 2007).

\section{El sector social de la vivienda en Europa}

Lo cierto es que, más allá de la actual crisis económica, la política social de vivienda ha estado sujeta a constantes variaciones debidas, fundamentalmente, a la diversificación de estrategias públicas existentes dentro de la Unión Europea. Estas diferentes aproximaciones nacieron, entre otras, en base a los tipos de tenencia y el 
grado de ayuda financiera pública, diferencias que se mantienen hasta la actualidad.

Las políticas de vivienda de protección oficial comenzaron a principios del siglo XX en Europa Central y del Norte, con medidas directas e indirectas para asegurar el acceso a una vivienda digna y de calidad a los ciudadanos. La parte más importante de esta fase en términos cuantitativos tuvo lugar después de la Segunda Guerra Mundial, en la que una gran cantidad de viviendas fueron construidas con la ayuda de prestaciones públicas. Esto ocurrió a través de estrategias diferentes según el país desde el que analicemos la intervención. En el Reino Unido y los Países Bajos, la vivienda de protección oficial fue construida y gestionada sobre todo por las autoridades locales; en Francia y Alemania, a través de delegaciones o empresas del sector público o privado, pero siempre bajo control público. En la mayoría de países europeos nórdicos, dicha intervención pública fue considerada una intervención para la regulación del mercado y para su reequilibrio, desarrollando medidas que afectaran a todas las categorías sociales y no únicamente a las clases más desfavorecidas (Roma, 2007).

En los países europeos del sur, el objetivo fundamental ha sido proporcionar ayuda para permitir a las familias comprar un hogar, en detrimento del alquiler. En este contexto, el porcentaje del gasto en vivienda dentro del gasto total del bienestar ha sido (y sigue siendo) tradicionalmente bajo, por lo tanto el papel de la vivienda de alquiler social es absolutamente marginal, siendo reservado, por lo menos en teoría, a las familias más pobres y marginadas.

Esta diferencia en los modelos de gestión hace que, dentro de los países de la Unión Europea, la población que reside en los parques sociales de vivienda sea muy variada. En este sentido, conviene tener en cuenta que, hasta la mitad de los años 80, no existían restricciones económicas para el acceso a una vivienda social en algunos países nórdicos, contando con una presencia relativamente importante de hogares situados en la media superior de la escala global de renta (Trilla, 2001).

Mientras tanto, en el caso de los países de Europa del Sur, el rol atribuido a las familias en el sistema de bienestar mediterráneo, ha caracterizado negativamente las políticas de vi- vienda. Esta considerable movilización de recursos y de redes familiares, junto con las ayudas públicas para la compra de la vivienda, ha permitido alcanzar altas tasas de propiedad privada (en referencia a la residencia principal), especialmente en España e Italia (Roma, 2007, p. 17) en detrimento de viviendas en régimen de alquiler privado $\mathrm{y}$, más aún, en viviendas de alquiler social.

Para ayudarnos a entender esta evolución en las políticas de vivienda, y las consiguientes consecuencias que han llegado a nuestros días en relación a la degradación de los barrios, las características de la población residente en estos parques de viviendas públicas, o la escasez de oferta de viviendas sociales para aquellas personas que sufren la crisis financiera y económica actual, es importante hacer referencia a los modelos de gestión y regulación de la política social de vivienda.

Siguiendo a Lachambre (citado en Trilla, 2001), en la perspectiva histórica sobre los modelos de gestión de las políticas de vivienda, se pueden observar dos estrategias diferenciadas. En primer lugar, el denominado «sector político» donde los parques sociales de vivienda se han abstraído de las realidades económicas en las diferentes etapas financieras. Su finalidad ha sido ofrecer una vivienda de alquiler siguiendo criterios estrictamente políticos, sin ninguna referencia económica, fijando los precios de una forma arbitraria, pactada entre las partes, o con una visión política. Así, las cuantías de las rentas exigidas por el Estado se encontraban, en la mayoría de las ocasiones, muy por debajo de la media del mercado y quedaban fijados al margen de los ingresos familiares. Este es el caso de Reino Unido (hasta 1988), Portugal, Irlanda, Luxemburgo y España.

En segundo lugar podemos observar el «sistema económico», cuyos objetivos políticos se centran en encontrar un equilibrio entre alquileres y capacidad económica de la población residente, con la garantía de disponer, continuadamente, del parque cuantitativamente necesario. Esta modalidad corresponde a Alemania, Austria, Holanda, Dinamarca y Reino Unido (desde 1988).

Los actores que intervienen en ambos modelos también son diferentes. En el «sistema político» son sociedades que gestionan, como concesionarios, el parque de alquiler (Reino 
Unido, Irlanda y Luxemburgo) o instituciones públicas que dependen del Estado (Portugal), comunidades autónomas (España) o municipios (Italia). En el caso del «sistema económico» los actores son sociedades de vivienda sin ánimo de lucro, independientes de los poderes públicos o sociedades de vivienda, y con niveles de dependencia variables con respecto a los municipios (Trilla, 2001).

En los últimos diez años ambos sistemas han sido objeto de un profundo debate para hacer frente a los nuevos retos que debe afrontar el sector del alquiler social en Europa. En el sistema político se ha observado un fracaso global en la gestión y administración de los parques de vivienda, lo que ha llevado a, por un lado, vender las viviendas a los inquilinos para liberar al sector público de un patrimonio muy costoso de mantener y con altos índices de endeudamiento de los arrendatarios. Y, por otro, substituir la fijación política de las rentas de alquiler por unos mecanismos que garanticen un equilibrio de explotación, lo que supone un acercamiento de ambos sistemas. Mientras tanto, el sistema económico tampoco se ha mantenido ajeno a estos debates, sobre todo debido a los altos costes de cuotas pero, también, por las disfunciones en la gestión y composición social de sus residentes.

A lo mencionado anteriormente, y en relación con el sector social del alquiler, desde la década de 1980, se han sucedido reajustes progresivos en las políticas generales, caracterizadas por una reducción importante de la intervención pública; las ayudas estatales directas para el alquiler están reduciéndose, mientras que las ayudas para el acceso a la propiedad se incrementan. Modificaciones, en definitiva, que se están experimentando en la inversión, la forma de gestión, la fijación de los alquileres, políticas de venta y la propia evolución demográfica de los residentes (Trilla, 2001).

Este reajuste de políticas no sólo ha llevado a una reducción de los recursos asignados, sino también a una revisión del gasto público en el sector de la vivienda, con un cambio del tipo de construcción de viviendas y de las ayudas directas a las familias; ambos cambios dirigidos tanto para la compra como para el alquiler cuando el porcentaje de la renta de la familia no excede ciertos niveles (Roma, 2007).
Estos modelos de gestión han implicado la redefinición de estrategias públicas llevadas a cabo en el pasado $\mathrm{y}$, además, plantean nuevos desafíos en la política social de los estados miembros. Se trata de responder a nuevas necesidades sociales en materia de alojamientos con una intervención sobre la población residente en estos parques de viviendas sociales. Así, los problemas sociales y de ordenación urbana emergentes, y que han producido vecindades segregadas y conflictos urbanos son, actualmente, una preocupación para las autoridades públicas. Entre otras cuestiones, además de reproducir las características de un sistema político con una compleja solución a medio plazo y necesitar de una intervención pública de gestión integral que, como se ha dicho, venza la perspectiva sectorializada que ha caracterizado a la vivienda social en las últimas décadas.

\section{Algunos retos para la política de vivien- das sociales en Europa}

La situación de los parques sociales de vivienda gestionados por empresas públicas, municipios o por organizaciones no lucrativas, está, en la mayoría de las ocasiones, relacionadas con una transformación que las identifica con reductos de viviendas de baja calidad, con los inquilinos más pobres y malas condiciones de vida (Roma, 2007; Trilla, 2001). En algunas ciudades europeas, las dificultades referentes al acceso y la calidad de la vivienda han creado problemas sociales de segregación que llevan a la concentración de grupos sociales con bajos ingresos en zonas urbanas degradadas y problemáticas. Esta situación demanda la atención sobre la integración social de las minorías étnicas, así como sobre los problemas en barrios periféricos conformados, en muchas ocasiones, por promociones sociales de viviendas.

Las principales problemáticas detectadas en las aglomeraciones de viviendas sociales son, entre otras: a) mala gestión financiera y gasto público excesivo; b) mala conservación de los edificios; c) creación de sectores marginales de desempleados de larga duración y personas mayores; d) concentración creciente de colectivos con rentas bajas y trabajadores pobres; e) intervenciones públicas sectoriales; f) desaparición del tejido empresarial y comercial con difícil regeneración de los mismos; g) falta de 
recursos orientados a una intervención integral; etc. (Trilla, 2001; Mira, 2001).

En los últimos años, se está constatando en Europa un aumento de problemáticas alrededor de las viviendas sociales que, por su carácter local, y la heterogeneidad de estrategias existentes en los estados miembros, no acaban de encontrar respuestas adecuadas por parte de las políticas públicas tradicionales. Siendo contenidas con respuestas de intervención social, en la mayoría de las ocasiones, sectorializadas y sin criterios de modernización, calidad y evaluación de resultados (elementos rectores de los servicios sociales de interés general), dichas estrategias de actuación ignoran, en demasiadas ocasiones, los contextos de la política social en la que nos situamos como profesionales.

En este sentido sigue siendo habitual no reconocer la vivienda como un servicio social a pesar que, cada vez más, se trate de una necesidad social no satisfecha por el mercado para una parte de la población cada vez mayor, y con perfiles tradicionalmente no asociados a los recursos sociales de carácter asistencial.

El problema de la vivienda es complejo y con dificultades comunes en muchos de los Estados miembros. El descenso de la demanda de la vivienda familiar tradicional, la presencia cada vez mayor de familias monoparentales y hogares unipersonales, el envejecimiento de la población autóctona por una parte y el incremento de inmigrantes por la otra, son dos factores que demandan con fuerza un cambio de oferta. Finalmente, en las grandes ciudades, los precios del mercado inmobiliario (que en la última década, han experimentado un crecimiento constante en casi todos los países de la UE), no sólo han llevado a mermar la capacidad de consumo de los hogares, sino que también han incidido sobre temas sociales $\mathrm{y}$ de desarrollo (Roma, 2007).

Según el Informe sobre Población y Condiciones Sociales, elaborado por Eurostat, 30.000.000 personas en la Unión Europea sufrieron falta de la vivienda y espacio deficientes en relación a las condiciones de vida. En 2009, el 6,0 por ciento de la población de la Unión Europea sufrió privación de vivienda grave. Así, los problemas más frecuentes que inciden sobre las condiciones de habitabilidad de los individuos fueron el ruido desde el ba- rrio (22,2 por ciento), el hacinamiento $(17,8$ por ciento) y la contaminación, suciedad u otros problemas ambientales (16,5 por ciento). Además, el 12,2 por ciento de las personas en la Unión Europea vivía en hogares afectados por el alto costo de la vivienda (Rybkowska y Schneider, 2011).

Como se indica en la Comunicación de la Comisión sobre Plataforma Europea contra la Pobreza y la Exclusión Social, el acceso al alojamiento es una necesidad fundamental. Sin embargo, los gastos relacionados con la vivienda a menudo representan una alta proporción de los hogares, lo que significa que la renta disponible podría impedir que algunas personas puedan satisfacer esta necesidad. La «tasa de costo de la vivienda» se define como el porcentaje de la población que vive en hogares donde el costo total de la vivienda supera el 40 por ciento de sus ingresos disponibles. Los costos de vivienda son la hipoteca o préstamos para la vivienda, el pago de intereses para los propietarios y pagos de alquiler para los inquilinos, servicios públicos (agua, electricidad, gas y calefacción) y los gastos relacionados con el mantenimiento regular y seguro estructurales también están incluidos. En 2009, el 12,2 por ciento de la población europea vivía en un hogar que gasta más del $40 \%$ de sus rentas disponibles equivalentes en materia de vivienda (idem).

Además de los datos cuantitativos que dibujan una perspectiva compleja para la vivienda en Europa, la política de vivienda en los estados miembros está sujeta a numerosas directrices, incluyendo la expansión de los mercados cada vez menos regulados, la merma y «residualización de las viviendas sociales» (Malpass, 2007 , p. 53) y la consiguiente disminución de la red de ayudas sociales. Esta tendencia en vivienda, en el contexto del proceso de reestructuración del Estado de Bienestar, ponen de manifiesto, por un lado, que las actuales medidas sobre las viviendas son insuficientes $\mathrm{y}$, por otro, permite comprender cómo el sector de la vivienda social se encuentra relacionado con la transformación y modernización del Estado de Bienestar.

La situación de debilidad de la política de vivienda se suma a otros factores que están poniendo a prueba la sostenibilidad del Modelo Social Europeo hasta el punto de re-pensar que 
la concepción del Estado de Bienestar como sistema en el que el Estado se reponsabiliza de proveer determinados servicios sociales está entrando en crisis. Una realidad que evidencia que, en la actualidad, los regímenes sociales europeos no sólo tienen defectos y debilidades inherentes, sino que están sufriendo dificultades para poder resolver algunos problemas reultantes de tendencias endógenas y exógenas (González, 2011).

Los desafíos a los que se enfrenta la política de vivienda en la Unión Europea son diversos. A pesar que la vivienda desempeña un papel fundamental en la vida de las personas, representando el gasto más importante en la mayoría de los hogares, y su localización fisica determina el acceso a bienes y recursos, está dejando de ocupar un lugar prioritario en la intervención pública progresivamente.

Aunque la vivienda ha sido uno de los pilares del Estado de Bienestar en muchos países europeos $\mathrm{y}$, desde los últimos años, se ha comenzado a contemplar como un servicio social de interés general, lo cierto es que está siendo objeto de una tendencia del Estado a reducir la provisión de las mismas y a transmitir las viviendas sociales a sus ocupantes debido al alto coste económico que suponen (Malpass, 2007). Asímismo, los gobiernos o entes públicos encargados de la política de vivienda se están viendo obligados, simultaneamente, a resolver cuestiones relacionadas, cada vez más, con la población que residen en los parques de viviendas sociales y las situaciones de exclusión y segregación que soportan en demasiadas ocasiones. Resolver modelos de gestión que no han funcionado, atender a la creciente demanda de viviendas sociales, contemplar la vivienda como un servicio social de interés general o vencer la mirada segregada de la vivienda en las intervenciones que se realizan son, por tanto, algunos de los retos a los que se enfrenta la política de vivienda, el Estado de Bienestar de muchos países de la Unión Europea y los profesionales que desarrollan su actividad en este ámbito.

Por tanto, estas situaciones emergentes, y de carácter estructural, demandan no sólo actuaciones de intervención social en barrios periféricos, parques de viviendas sociales de alquiler $\mathrm{y}$, en muchas ocasiones, cascos históricos que comparten problemas sociales con las aglome- raciones de alojamientos sociales. Se trata de contemplar la posibilidad de visibilizar la vivienda social como un servicio esencial para los ciudadanos a través de actuaciones que deben, en la medida de lo posible, considerar las cuestiones de la política social, los debates en materia de inversión pública o las estrategias comunes elaboradas en una coyuntura económica como la actual para, en la medida de lo posible, evitar reproducir respuestas sectoriales que no provocan las transformaciones sociales inclusivas adecuadas.

En este sentido, la reflexión de los profesionales vinculados, de una manera u otra, a las políticas sociales en materia de vivienda resulta fundamental. Se trata de poseer una mirada compleja, más allá de respuestas prestacionales enfocadas, prácticamente con exclusividad, a modelos tradicionales de gestión económica o política en el sector social de la vivienda, centradas en prestaciones económicas o procesos parciales de rehabilitación urbana. Así, integrar una visión micro y macro social, formar parte de la elaboración de estrategias de actuación o la planificación de procesos, requiere, entre otras cuestiones, conocer los contextos supranacionales y, en ocasiones, vencer el reduccionismo que nos lleva, en demasiadas ocasiones, a hacer nuestros los intereses de índole institucionalistas y no técnicos. Convirtiendo estos intereses en las respuestas profesionales.

En definitiva, los servicios sociales de interés general, con la vivienda social contemplada como servicio esencial, requieren para su modernización y adecuación a la situación actual, superar el concepto tradicional entendido como actividad prestacional de carácter económico para ser reemplazado por un nuevo concepto en el que la idea central sea la asunción, por las Administraciones, de la responsabilidad de la garantía de determinadas prestaciones técnicas (Tornos y Galán, 2007).

\section{Conclusiones}

Desde el año 2000, cuando se aprobaron la Estrategia de Lisboa y los sucesivos documentos generados por la Unión Europea, los servicios sociales de interés general se han incorporado como eje que favorece la dimensión social del llamado modelo social europeo. Un acontecimiento que ha favorecido una mayor visualización de los servicios sociales en el espacio co- 
munitario y su consideración como esenciales en la persecución de la cohesión social. La educación, los cuidados de larga duración, las ayudas directas a los individuos fuera de los sistemas de cotización y la vivienda social, entre otros, han sido reconocidos como elementos que generan menor desigualdad social, siendo fundamentales en la lucha contra la pobreza y la exclusión social.

Si bien es cierto que los Estados miembros se encuentran en una etapa de replanteamientos sobre modelos de gestión pública de los problemas sociales, no es menos cierto que, en función de las tendencias en materia de servicios sociales como servicios esenciales e integrales, las intervenciones profesionales deben, cada vez más, tener en cuenta qué está sucediendo con las políticas sociales, hacia dónde vamos o cómo las tendencias a nivel macro/supranacional pueden condicionar las acciones en los barrios, en las comunidades, en nuestras ciudades.

En este sentido, los debates sobre política social parecen, todavía, ajenos a algunas profesiones como el trabajo social, centradas en los análisis de procesos microsociales o en intervenciones de carácter sectorializado de determinados grupos de población o de problemas sociales específicos. Una visión de los Servicios Sociales que pueden impedir, entre otras cuestiones, observar la integralidad necesaria que se da en los servicios sociales esenciales que incluyen, como hemos podido ver, la educación, la vivienda social o los cuidados de larga duración. En definitiva, una mirada sistémi- ca u holística de los servicios sociales de interés general al margen de la esfera económica o de producción de bienes.

El reto para profesionales de la intervención social en materia de política de vivienda es, cada vez más, poseer la capacidad de análisis que nos permita hermanar el desarrollo comunitario con el conocimiento de estrategias que posibiliten superar la gestión administrativa, teniendo como horizonte los derechos sociales y la justicia social, venciendo la visión de gestión administrativa que, cada vez más, abstraen a los servicios sociales en general y sus profesionales de la acción en el territorio. Haciendo nuestras estrategias basadas en modelos de gestión económicos o políticos que, como hemos podido observar, continúan teniendo consecuencias inadecuadas para los ciudadanos y los propios estados.

Se trata pues de no abandonar el análisis de los problemas sociales, de las políticas sociales, de los sistemas de protección. Se trata de continuar creyendo en un sistema universal de servicios sociales que venzan la actual segregación de intervención. Se trata de evitar un distanciamiento entre las profesiones de la intervención social y las reflexiones que requieren la actual situación de las políticas sociales y los instrumentos que, desde ella, se plantean como respuestas técnicas y prestacionales. Instrumentos que los trabajadores sociales, entre otros profesionales, nos encargamos de materializar en los procesos de intervención con la ciudadanía.

\section{Referencias bibliográficas}

Comunicación de la Comisión al Parlamento Europeo, a. C. (2004). Libro Blanco sobre los servicios sociales de interés general. (B. C. Europeas, Ed.) Recuperado de: http://eurlex.europa.eu/LexUriServ/site/es/com/2004/com2004_0327es01.pdf.

Comisión, C. d. (2006). Aplicación del programa comunitario de Lisboa. Servicios sociales de interés general en la Unión Europea. (B. C. Europeas, Ed.) . Recuperado de: http://eur-lex.europa.eu/LexUriServ/site/en/com/2006/com2006_0177en01.pdf.

Consejo de Ministros de Desarrollo Urbano y Cohesión Territorial de la Comunidad Europea de Naciones (mayo, 2007). Carta de Leipzig sobre Ciudades Europeas Sostenibles. Recuperado de: http://www.mviv.es/es/pdf/AGENDA_URBANA/AUE_ENE09_leipzig.pdf.

Consejo de la Unión Europea (2009). Informe conjunto sobre protección social e inclusión social. Bruselas. Consejo (Empleo, Política Social, Sanidad y Consumidores). Recuperado de: http://eur-lex.europa.eu/LexUriServ/LexUriServ.do?uri=COM:2009:0058:FIN:ES:PDF.

De Rossa, P. (2009). Informe sobre el futuro de los servicios sociales de interés general. Bruselas: s.n. Recuperado de: http://www.europarl.europa.eu/sides/getDoc.do?type=REPORT\&reference $=\mathrm{A} 7-2011-0239 \&$ language $=\mathrm{ES}$. 
González Vázquez, I. (2011). El cambio de orientación de la política social comunitaria. Del Estado de Bienestar a la Sociedad del Bienestar (Tesis inédita). Sevilla: Universidad de Sevilla. Recuperado de: http:// fondosdigitales.us.es.

Malpass, P. (2007). La transformación del Estado de Bienestar y la vivienda: consecuencias desde la perspectiva británica. Transformaciones del Estado de Bienestar y cambios demográficos en Europa: Retos del sector de la vivienda social (pp. 53-69). Barcelona: CECODHAS. Recuperado de: http://www.promotorespublicos.org/public/ficheros/publicaciones/ 5a7183fa54e72a19b0b8bea49d8229ae.pdf.

Mira, F. J. (2001). Territorio, hábitat y vivienda frente a la exclusión social. Alternativas. Cuadernos de Trabajo Social, 9, 241-262. Recuperado de: http://hdl.handle.net/10045/5735.

Roma, G. (2007). Transformación del Estado del bienestar en Europa: análisis comparativo. Transformaciones del Estado de Bienestar y cambios demográficos en Europa: Retos del sector de la vivienda social (pp. 14-24). Barcelona: CECODHAS. Recuperado de: http://www. promotorespublicos.org/public/ficheros/publicaciones/5a7183fa54e72a19b0b8bea49d8229ae. pdf.

Rybkowska, A., y Schneider, M. (2011). Housing conditions in Europe in 2009. Recuperado de «Income, Social Inclusion and Living Conditions»: http://epp.eurostat.ec.europa.eu/portal/page/portal/income_social_inclusion_living_conditions/data/database.

Tornos Mas, J. y Galán Galán, A. (2007). La configuración de los servicios como servicio público. Derecho subjetivo de los ciudadanos a la prestación del servicio. Madrid: Ministerio de Trabajo y Asuntos Sociales. Secretaría de Estado de la Seguridad Social. Recuperado de: http://www.seg-social.es/prdi00/groups/public/documents/binario/51598.pdf.

Trilla, C. (2001). La politica de vivienda en una perspectiva europea comparada., Madrid: Fundación La Caixa. Colección Estudios Sociales 9. Madrid. Recuperado de http://obrasocial.lacaixa.es/StaticFiles/StaticFiles/3409195ca52ef010VgnVCM1000000e8cf10aRCRD/es/es09_i nx_es.pdf. 